\title{
Los protocolos universitarios para la prevención y sanción de la violencia, acoso y discriminación entre estudiantes: una mirada criminológica y político-criminal
}

\author{
José Ángel Fernández Cruz*
}

\begin{abstract}
RESUMEN
El presente trabajo reconstruye los protocolos universitarios contra el acoso, la violencia y la discriminación entre estudiantes desde consideraciones criminológicas y político-criminales. Así, desde la criminología, podemos destacar la autonomía universitaria como uno de los factores que ha favorecido una cultura de la impunidad y, desde la política criminal, estos protocolos constituyen una de las principales manifestaciones de reconocimiento de los derechos de las víctimas.
\end{abstract}
Violencia - acoso y discriminación en las universidades - autonomía universitaria - victimología

\section{Chilean Policies and Procedures on Violence, Harassment and Discrimination among Students. Perspectives from Criminology and Criminal Justice}

\begin{abstract}
The present study provides a criminology and criminal justice analysis of Chilean universities' policies and procedures on violence, harassment, and discrimination among students. From criminology considerations, it should be noted that autonomous university has facilitated a culture of impunity of these types of conducts. From criminal justice perspective, university policies and procedures on violence, harassment and discrimination is one of the principal manifestations of recognition of victims' rights.
\end{abstract}

Violence - harassment and discrimination on campus - autonomous university - victimology.

* Licenciado en Derecho, Universidad Complutense de Madrid, España. Doctor en Derecho, Universidad Europea de Madrid, España. Profesor de Derecho Penal, Universidad Austral de Chile. Correo electrónico: josefernandez@uach.cl

Artículo recibido el 5.3.2020 y aceptado para su publicación el 4.7.2020. 


\section{INTRODUCCIÓN}

$\mathrm{L}$ a problemática de la violencia, acoso y discriminación en las universidades (en lo sucesivo, AVD) ha sido una cuestión que, hasta tiempos recientes, había recibido poca atención no solo respecto de las autoridades estatales y universitarias, sino también por parte de la academia. Sin embargo, en los últimos años y como consecuencia de las movilizaciones feministas, varias universidades han aprobado protocolos internos destinados a prevenir y sancionar este tipo de $\operatorname{conductas}^{1}$, normativa que, por su complejidad, requiere de una reflexión académica.

El presente trabajo realizará una reconstrucción de estos protocolos a partir de la criminología y la política criminal. La pertinencia de este enfoque se debe, entre otras razones, al hecho de que estos protocolos internos abordan el objeto por antonomasia de las ciencias penales, a saber, la violencia.

La tesis fundamental de este estudio es que una mirada desde las ciencias penales permite comprender y, en último término, legitimar estos protocolos, ya que uno de los principales problemas que se ha detectado, al menos en el Reglamento UACH, es una incomprensión y cierto rechazo por parte de las partes involucradas y de la comunidad universitaria. Para ello, este trabajo se sustenta, en la parte criminológica, en una visión crítica y realista de la violencia y, en la parte político-criminal, en una propuesta bienestarista penal centrada en la reinserción y en una visión no punitivista de reconocimiento de los derechos de las víctimas. Así, en cuanto a las cuestiones criminológicas, el estudio se centra en la violencia de género entre estudiantes, en las consecuencias expresas o latentes de los protocolos y en explicar las altas tasas de impunidad en el seno de las universidades. Respecto de la reconstrucción político-criminal, el estudio propone, en último término, un papel más activo de las universidades a la hora de sancionar y prevenir la violencia mediante una extensión de su competencia territorial y, a la vez, aboga por una redacción e interpretación de estos protocolos que reconozca los derechos tanto de las víctimas, por medio de medidas de protección y acompañamiento y soluciones alternativas, como de los estudiantes mediante un debido proceso, una sanción proporcionada y unas medidas de reinserción.

Este estudio toma como referencia el Reglamento, aprobado el 26 de junio de 2016, de "Procedimiento para el Acompañamiento, Investigación y Sanción de Conductas de Acoso, Violencia y Discriminación entre los Estudiantes de la Universidad Austral de Chile" (en lo sucesivo, Reglamento UACH) ${ }^{2}$, ya que ha sido la primera universidad del

${ }^{1}$ En la actualidad, aproximadamente 20 universidades chilenas cuentan con protocolos relacionados con la violencia, acoso o discriminación: Alberto Hurtado, Metropolitana de la Educación, Arturo Prat, Antofagasta, La Serena, Católica de Valparaíso, Los Lagos, Magallanes, Diego Portales, de la Frontera, de Chile, O’Higgins, Católica de Chile, Valparaíso, Tecnológica Metropolitana, Santiago de Chile, Concepción y Tarapacá.

${ }^{2}$ En el año 2015, la UACH aprobó la "Política de Prevención y Sanción del Acoso, Violencia y Discriminación en la Comunidad Universitaria”. Ese mismo año, se creó el Comité Paritario de Género y Diversidad, constituido por representantes de los distintos estamentos de la universidad, cuyo objetivo fundamental es "promover la inclusión de la perspectiva de género y diversidad a través de la proposición de 
país en aprobar un reglamento que regula de manera expresa los casos de AVD entre estudiantes. También se prestará especial atención a la literatura criminológica estadounidense, ya que ha sido en este país donde se han desarrollado e investigado con mayor profusión estos protocolos.

\section{UNA BREVE RESEÑA AL REGLAMENTO UACH}

La promulgación del Reglamento UACH, al igual que en el resto de las universidades chilenas, ha supuesto un cambio estructural en la manera que se aborda la problemática del AVD, no solo respecto de los procedimientos disciplinarios y del diseño institucional, sino también en cuanto a las prácticas de la comunidad universitaria.

El Reglamento UACH comienza estableciendo su ámbito de aplicación, en el que se incluye no solo el AVD realizado dentro del campus universitario o en el contexto de actividades universitarias, sino también aquellos casos fuera de estos supuestos calificados como graves y necesitados de una especial protección de la víctima (Art. 1).

Respecto de las conductas o hechos tipificados, el Reglamento UACH establece tres grandes categorías: el acoso, la violencia y la discriminación. Respecto del primero se diferencia entre el acoso sexual, el discriminatorio y el psicológico. En cuanto a la violencia, se distingue entre la de género y la discriminatoria. Y, por último, la discriminación hace referencia a toda distinción, exclusión o restricción que carezca de justificación razonable, en particular cuando se funden en motivos como la raza o etnia, ideología, orientación sexual o identidad de género (Art. 2).

En cuanto a la parte orgánica o institucional, el Reglamento UACH contempla tres organismos. Dos internos: la Comisión para Situaciones de Acoso y Violencia y Discriminación (en lo sucesivo, la Comisión) y la Secretaría Ejecutiva (en lo sucesivo, la Secretaría); y uno externo de apoyo, la Dirección Jurídica de la Universidad. La Comisión es un órgano colegiado compuesto, entre otros, por un jurista, un especialista en género y diversidad y un representante de los estudiantes. Respecto de los otros miembros, se ha privilegiado el nombramiento de expertos en el tratamiento del AVD (psicólogos y asistentes sociales). En este sentido, debemos señalar que el Reglamento UACH presta una especial atención no solo a la protección y bienestar de las víctimas, sino también al tratamiento y resocialización del infractor. Por tanto, la protección de la víctima y la reinserción del victimario justifican la composición mixta de este órgano. Sus principales funciones son recibir y admitir las denuncias o reclamaciones, adoptar las medidas de protección y acompañamiento que contempla el Reglamento UACH y resolver los procedimientos disciplinarios (Art. 3 a 5). La Secretaría ejerce las labores de coordinación

políticas, procedimientos, acciones y metas (...)". Decreto No 76 de 15 de diciembre de 2015 . A continuación se promulgó en el 2016 el Reglamento UACH. Finalmente, en el 2018 se aprobó el "Reglamento de Intervención en Situaciones de Acoso, Violencia y Discriminación”, que abarca el resto de los posibles casos de AVD que pueden darse dentro de la comunidad universitaria, en especial entre estudiantes y profesores y entre profesores y personal administrativo. 
de la Comisión, aunque sin derecho a voto. Tiene como principales funciones: recibir las denuncias por parte de los estudiantes, asesorar a estos acerca del Reglamento y exponer los antecedentes a la Comisión a la hora de admitir la tramitación de un procedimiento

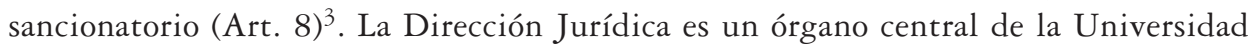
que tiene como cometido la instrucción de los procedimientos disciplinarios (Art. 18).

El procedimiento se divide en tres grandes fases: el inicio y admisibilidad de la denuncia, la instrucción y la resolución. Además, el procedimiento en todas sus etapas está informado por ciertos principios y garantías generales consagradas en el artículo 11 del Reglamento. Así, tenemos:

a) El principio de buena fe de las personas implicadas en el esclarecimiento de los hechos denunciados. Este principio viene acompañado de la posibilidad de incoar un procedimiento disciplinario en los casos de la utilización manifiestamente maliciosa de este procedimiento;

b) El principio de objetividad en la investigación para determinar la existencia de los hechos denunciados;

c) Los principios de dignidad, intimidad e igualdad durante la tramitación del procedimiento;

d) La garantía del tratamiento reservado de la información relativa a las situaciones que pudieran ser constitutivas de los hechos que regula este Reglamento;

e) El principio de protección a las víctimas, que establece un criterio abstracto de ponderación a favor de estas. Así, “cada vez que sea necesario realizar ajustes o cambios de cualquier tipo, se buscará que la parte ofendida o agredida soporte la menor parte de estos, así como también se le otorgará el máximo posible de seguridades y de facilidades en la realización de la denuncia, la investigación y la resolución de esta”.

En cuanto al inicio del procedimiento, el Reglamento establece la obligatoriedad de todos los órganos directivos como, por ejemplo, los directores de escuela de poner en conocimiento de la Comisión cualquier hecho que sea susceptible de ser considerado como AVD. Iniciado el procedimiento la víctima podrá desistir de la denuncia, lo que conllevará el fin de este, salvo hechos calificados como graves por la Comisión (Art.13). En todo caso, el desistimiento de la víctima no es obstáculo para que la Comisión pueda adoptar medidas de acompañamiento (art. 14).

La etapa de admisibilidad contempla tres posibles soluciones: (1) la inadmisión de la denuncia en sentido estricto; (2) la inadmisión de la denuncia acompañada de una

${ }^{3}$ Además, debemos añadir, con posterioridad a la entrada en vigor del Reglamento UACH, la creación de la Unidad de Acompañamiento para Casos de Acoso, Violencia y Discriminación contra Estudiantes. Su creación se hizo necesaria debido al hecho de que una parte importante de las consultas recibidas por la Secretaría eran hechos que afectaban a estudiantes de la Universidad, en los que el presunto agresor no era otro estudiante o se encontraban fuera de la competencia de la Comisión. Esta Unidad se centra en prestar atención psicológica, orientación jurídica y eventuales beneficios sociales. 
línea de acción dirigida a poner fin a la situación denunciada y evitar que se repita en el futuro; y (3) la admisión de la denuncia e inicio de la instrucción del procedimiento disciplinario. En este último caso, el Reglamento UACH contempla la posibilidad de aplicar medidas de protección y acompañamiento.

El procedimiento disciplinario es instruido por un abogado de la Dirección Jurídica de la Universidad. Se debe destacar un notable esfuerzo en medios y formación de esta unidad en la instrucción de este tipo de casos y, en especial, en aquellos relacionados con la violencia de género o machista. También, debemos destacar que la comparecencia de los testigos es obligatoria. Por último, tanto la víctima como el denunciado podrán ser acompañados por terceras personas y, en especial, de abogados (Art. 18).

El Reglamento contempla la suspensión del procedimiento en el caso de que se inicie un procedimiento penal, sin perjuicio de aplicar o mantener alguna de las medidas de protección o acompañamiento (Art. 15). En todo caso, la terminación del proceso penal sin condena (sobreseimiento, suspensión condicional, absolución, etc.) no cierra la posibilidad de que el hecho sea sancionado por el Reglamento UACH.

Por último, la resolución del procedimiento, como hemos mencionado, compete a la Comisión. Esta resolución se articula a partir de una comprensión argumentativa de las decisiones judiciales. Así, esta debe contener los hechos investigados, las pruebas remitidas, el resumen de las alegaciones planteadas por las partes, la decisión adoptada por la Comisión, debidamente fundada, y, en su caso, la sanción aplicable (art. 19).

\section{Cuestiones CRIMINOLÓGICAS}

\section{Una cuestión de género y violencia sexual}

El AVD en el ámbito universitario ha sido un fenómeno social silenciado hasta fechas recientes. Esta situación ha sido especialmente grave en los casos de violencia sexual contra las mujeres y los sujetos no conformes con su género ${ }^{4}$. La violencia en las universidades es un problema que afecta principalmente al género femenino y se centra en conductas de connotación sexual. Desde el estudio de Koss, Gidycz y Wisniewski (1987), en el que se evidenció que 1 de cada 4 estudiantes universitarias estadounidenses habían experimentado algún tipo de violencia sexual desde los 14 años, existe una constante y sólida evidencia empírica respecto del hecho de que las mujeres son las principales víctimas de la violencia en las universidades ${ }^{5}$.

\footnotetext{
${ }^{4}$ Mellins et. al., 2017, p. 3 y doctrina citada.

${ }^{5}$ En Estados Unidos, véanse los estudios citados por PERKins y Warner, 2017, p. 237. No obstante, Beaver critica estos estudios por incompletos y metodológicamente incorrectos. Esto ha llevado al autor citado a afirmar que, a partir de estas investigaciones, el gobierno federal estadounidense ha implantado ciertas políticas que han supuesto un alto coste económico para las universidades y una merma de las garantías procesales (BEAVER, 2017, pp. 257-266). Respecto de la UACH, en los años 2016 y 2017, la gran mayoría de las consultas recibidas por la Secretaría se refieren a hechos en donde las víctimas son mujeres $(90 \%)$ y
} 
Además de los efectos directos e indirectos derivados de los propios actos de AVD, estos afectan al normal desarrollo de la formación académica de los estudiantes. Así, por ejemplo y respecto de las agresiones sexuales -además de la lesión a la libertad sexual, la integridad personal y la vida; infecciones de trasmisión sexual; embarazos no consentidos; abuso de sustancias psicotrópicas y alcohol; riesgo de suicidio; aislamiento social y disfunciones sexuales- estas conductas afectan gravemente al normal desarrollo de la formación académica de los estudiantes ${ }^{6}$. Estos efectos pueden agravarse considerablemente cuando la víctima se encuentra en el primer año de la universidad, ya que en este período los estudiantes, al tener que adaptarse a la nueva vida universitaria, se encuentran especialmente vulnerables ante cualquier acción violenta no consentida ${ }^{7}$. En este sentido, la violencia sexual contra las mujeres incide negativamente en las tasas de deserción universitaria. Así, en Estados Unidos, los estudiantes de primer año que han sufrido un acto de violencia tienen un mayor impacto en su desarrollo académico y son más proclives a dejar la universidad ${ }^{8}$.

Entre los factores de riesgo detectados por la criminología comparada podemos destacar la ingesta de alcohol o drogas 9 , las fiestas universitarias, las residencias de estudiantes, las relaciones sexuales causales y la pertenencia al primer año universitario. En Estados Unidos, el consumo de alcohol está asociado, al menos, con la mitad de los casos de agresiones sexuales. Esto indica que estamos ante uno de los factores más relevantes. En este sentido, la mitad de los estudiantes universitarios estadounidenses consumen alcohol de manera excesiva ${ }^{10}$. Como manifiesta Beaver, el consumo de alcohol es parte de la cultura universitaria, por lo que los problemas asociados a este continuarán en el futuro ${ }^{11}$. En el mismo sentido, también las hermandades universitarias crean un contexto sociocultural, en el que las relaciones sexuales no consentidas se encuentran normalizadas $^{12}$. En Chile, las fiestas universitarias, los hogares estudiantiles, las casas y departamentos compartidos por estudiantes y las actividades asociadas a la bienvenida de estudiantes de primer año (el mechoneo), unido con el consumo de alcohol y drogas constituyen, al menos, en la $\mathrm{UACH}$, los contextos socioculturales universitarios que

a quienes se les imputan las conductas que motivan las consultas son mayoritariamente hombres $(67 \%)$. Respecto de las conductas denunciadas, corresponden, mayoritariamente, a los tipos de violencia de género (36\%). Las mujeres son quienes denuncian con mayor frecuencia ser víctimas de AVD, representando el $65 \%$ de los estudiantes denunciantes. Si tomamos las denuncias individuales excluyendo, por tanto, las hechas por o contra grupos de estudiantes (véanse los casos de mechoneo), el $81 \%$ de las denunciantes fueron mujeres y el $80 \%$ de los denunciados hombres. En el año 2018, el sesgo de género resulta en términos porcentuales más evidente MEMoria 2016-2018.

${ }^{6}$ Véanse, entre otros, LÉvesQue et al., 2016.

${ }^{7}$ Por todos, Mengo y Black, 2015, p. 2.

${ }^{8}$ Mengo y Black, 2015, pp. 3-13.

${ }^{9}$ Mellins et al., 2017, pp. 1, 6, y 24. El uso de alcohol y otras drogas no solo debe ser visto en el contexto de un uso consentido por ambas partes, sino también como una estrategia deliberada para incapacitar a la víctima o limitar su posible resistencia. LisaK y Miller, 2002, pp. 73-84.

${ }^{10}$ National Institute on Alcohol and Alcoholism, 2014.

11 BeAver, 2017, p. 264.

12 Martin y Hummer, 1989, p. 462. 
contribuyen en mayor medida a conductas violentas en general y a relaciones sexuales no consentidas en particular.

Una de las dificultades a la hora de cuantificar el AVD en la comunidad universitaria radica en el hecho de que bajo las normas definitorias se han incluido conductas que difieren en cada uno de los protocolos ${ }^{13}$. Esto puede distorsionar la percepción que tiene la sociedad acerca de esta problemática. Como manifiesta Beaver, a la hora de cuantificar el AVD en las universidades, un factor determinante es cómo estas se encuentran definidas ${ }^{14}$. Así, por ejemplo, cuando utilizamos el término "relaciones sexuales no consentidas" para cuantificar, prevenir y sancionar una conducta desviada, corremos el riesgo de equiparar valorativamente un beso forzado con una violación ${ }^{15}$. Esta situación se da en Chile con el término acoso que comprende conductas que van desde un insulto en las redes sociales hasta el denominado acoso sexual quid pro quo.

Estos protocolos tienen como una de sus principales virtudes la pretensión de reducir las tasas de actos violentos no denunciados ante las instituciones penales, aunque debemos tener presente que las tasas de delincuencia no detectadas en las universidades son altas. Así, una hipótesis planteada en la literatura estadounidense, en el caso de la violencia sexual, mantiene que gran parte de los estudiantes que ha experimentado un acto de violencia o acoso sexual no lo ha percibido como tal, porque lo asocian a un acto violento físico realizado por un extraño, en contraposición con los casos en los que han concurrido previamente un acto de consentimiento en el que participa un conocido (compañero universitario o sentimental) ${ }^{16}$. Ahora bien, y como consecuencia de las movilizaciones universitarias chilenas, se percibe un cambio cultural a la hora de denunciar actos de violencia sexual cometidos por un compañero.

Especial relevancia en las universidades chilenas tienen, sin duda, los casos de violencia en el contexto de relaciones sentimentales entre estudiantes, comúnmente denominada como "violencia en el pololeo" ${ }^{17}$. La sanción de este tipo de violencia resulta especialmente problemática en el derecho chileno, ya que no existe hasta la fecha ninguna regulación estatal que la contemple de manera expresa ${ }^{18}$. De esta manera, los protocolos de AVD pueden erigirse con un instrumento idóneo y eficaz ante este tipo de conductas. Pero como veremos con posterioridad, recientes fallos de los tribunales de justicia han limitado la posibilidad de que estos protocolos conozcan casos de AVD acaecidos fuera de las instalaciones de la universidad o de contextos académicos.

${ }^{13}$ Esta situación se ha dado en las universidades estadounidenses a la hora de cuantificar las agresiones sexuales. Una de las razones por la cuales existen variaciones estadísticamente relevantes a la hora de cuantificar la violencia en las universidades reside en el hecho de que los protocolos incluyen diferentes definiciones. Así en el caso de las agresiones sexuales se incluyen conductas como el acoso sexual, tocamientos no consentidos, acoso callejero (tocamientos por sorpresa), entre otros. Mellins et al., 2017, p. 2.

${ }^{14}$ BeAver, 2017, p. 264.

${ }^{15}$ Incluso, es posible equiparar las penas en el derecho penal chileno. Véase, Fernández, 2015, pp. 443-458.

16 Fisher et al., 2010.

17 Poó y Vizcarra, 2008, pp. 81-88.

${ }^{18}$ Véase, el Proyecto que Modifica la Ley de Violencia Intrafamiliar y otros cuerpos legales y, establece Ley sobre Violencia en las Relaciones Íntimas de Pareja sin Convivencia, Boletín No 8851-18. 


\section{Consecuencias de los protocolos}

La criminología no solo se ocupa de los efectos y la etiología de la delincuencia, sino también de los asociados a los medios de control social, en especial, de los indeseados previstos o no. Por tanto, resulta necesario mencionar someramente algunas de las consecuencias de estos protocolos. Respecto de las víctimas, estos protocolos, al igual que en el sistema de justicia penal, pueden producir una revictimización como, por ejemplo, aquellos efectos asociados a interrogatorios hostiles, actitudes que denotan incredulidad respecto de la denuncia y a la percepción de no sentirse apoyadas por las instituciones universitarias ${ }^{19}$.

En cuanto a los denunciados y sancionados, el principal efecto radica en la merma de sus garantías procesales en aras de una mayor protección de las víctimas. Así, muchos de ellos se sienten desprotegidos y privados de sus derechos ${ }^{20}$. A lo anterior, debemos añadir los problemas asociados a la salud mental que conlleva el procedimiento sancionatorio y la eventual sanción. Pero, sin duda, las "funas" o "escraches" y juicios paralelos por medio de las redes sociales son unos de los efectos más perjudiciales de estos protocolos.

\section{La autonomía universitaria como espacio de impunidad}

La autonomía universitaria ha tenido un papel, en cierta manera, contradictorio en la prevención y sanción del AVD en las universidades. Por una parte, la inviolabilidad y el fuero universitario han impedido de forma expresa o latente que el Estado y las propias universidades intervengan en la prevención y sanción de la violencia en la comunidad universitaria; y, por otra, la autonomía académica, política, administrativa y económica ha permitido que varias universidades tomen medidas activas, entre la que destaca, precisamente, la promulgación de protocolos internos.

El fuero o autonomía universitaria en un sentido amplio comprende la libertad de enseñanza e investigación, el denominado fuero interno ${ }^{21}$ y la autonomía política, administrativa y económica. La autonomía universitaria ha formado parte de las universidades prácticamente desde su creación y se suele asociar a la defensa de intereses corporativos de los gremios. Con el tiempo, las universidades, a partir de una doble protección (primero pontificia y, con posterioridad, regia), se constituyen en unas corporaciones de amplia proyección con autonomía económica, administrativa y jurídica. Es así como se configuró como un organismo estructurado mediante varios privilegios frente al poder ${ }^{22}$.

\footnotetext{
${ }^{19}$ Harper et al., 2017, p. 306 y doctrina citada.

20 TripletT, 2012.

${ }^{21}$ El denominado fuero universitario, como un conjunto de reglas adscritas al principio de autonomía universitaria, comprende el mantenimiento del orden interno por parte de sus autoridades, la inviolabilidad de los recintos universitarios y, en sus inicios, la competencia exclusiva para conocer y juzgar a los miembros de la comunidad universitaria.

22 Ballbé, 1999, p. 4.
} 
Uno de los principales privilegios fue el denominado fuero académico, que suponía una competencia exclusiva de sus autoridades para juzgar a sus estudiantes y maestros ${ }^{23}$.

Pero con el advenimiento de las ideas ilustradas y, en especial, como consecuencia de los principios de libertad del trabajo y libertad económica y de una estructura centralista y absolutista del poder, se cuestionó y redujo la autonomía universitaria. No obstante, este cuestionamiento nunca supuso una eliminación total de estos derechos y prerrogativas e, incluso, con la consolidación de los modernos estados de derecho, se recuperaron gran parte de estos ${ }^{24}$. Ahora bien, en países como España y Chile, la dictadura supuso una reducción o eliminación de la autonomía universitaria. Hasta el advenimiento de la dictadura de Pinochet, se reconocían a las universidades tanto la garantía de no intromisión en materia de seguridad como la autonomía universitaria, situación que se interrumpe con el golpe de Estado de 1973. Por medio del Decreto Ley $\mathrm{N}^{\circ} 50$, del mismo año, la Junta Militar intervino en las universidades públicas y privadas, nombrando a sus autoridades con poderes absolutos y atribuyéndose la facultad para designar rectores delegados en todas las universidades del país ${ }^{25}$.

Tras el regreso de la democracia, se han recuperado de iure ${ }^{26}$ o de facto gran parte de los derechos y garantías asociados a la autonomía universitaria. Si bien el denominado fuero universitario no ha sido restablecido, se ha generado una cultura de no intervención, tanto de las fuerzas de seguridad del Estado como de la Fiscalía ${ }^{27}$, salvo en los casos de un delito flagrante o de seguridad pública. Así, nos encontramos con un concepto consuetudinario de domicilio universitario que impide o limita la intromisión de la policía y del Ministerio Público. Pero, además, las autoridades universitarias no han abordado los casos de AVD, especialmente, aquellos tipificados penalmente, ya que se ha entendido que su prevención, sanción y tratamiento correspondía a las diferentes instituciones del Estado. En conclusión, existía, hasta la promulgación de estos protocolos, un espacio y cultura de impunidad. Debemos recordar que, además de estas razones meramente competenciales, sin duda, el hecho de que la mayoría de los casos de AVD se encuentren relacionados con la violencia de género explica la invisibilización de estas conductas.

Si bien esta garantía de no intervención ha protegido ciertos derechos de los miembros de la comunidad universitaria, como el de libertad de expresión, también ha facilitado la vulneración de otros derechos, como la integridad física, la intimidad y la libertad sexual. En el tema que nos ocupa, esta cultura de la impunidad ha dejado sin protección a los estudiantes y, en especial, a las mujeres, invisibilizando este tipo de conductas no solo respecto de las autoridades académicas sino también y lo que es más

23 AbDelKader, 2011, p. 29.

${ }^{24}$ AbDelkader, 2011, p. 31.

${ }^{25}$ BASSA y Aste, 2109, p. 193 y 196.

26 Véase en extenso: BAsSA y Aste, 2109, pp. 195-199.

27 Véase un caso paradigmático de cómo esta prohibición se encuentra asentada en la cultura universitaria en: https://www.cooperativa.cl/noticias/pais/educacion/movimiento-estudiantil/por-que-carabineros-no-puede-ingresar-a-la-universidad-de-chile/2013-06-14/182516.html https://www.emol.com/ noticias/nacional/2012/03/29/533401/carabineros-le-responde-a-rector-zolezzi--tenemos-facultad-de-ingresoante-delitos-flagrantes.html 
chocante de los propios representantes estudiantiles que, hasta el año 2016, el AVD no formó parte de sus agendas políticas. Como manifiesta Caro, "si bien la autonomía es un valor fundamental, no puede ser en ningún momento una excusa para violar, vulnerar o esconder derechos" 28 . Así, no le falta razón a quienes critican la desnaturalización de la autonomía universitaria que, por una parte, ampara las prerrogativas de los profesores y de las instituciones universitarias y, por otra, permite que estas no se hagan cargo de sus responsabilidades sociales ${ }^{29}$.

\section{Cuestiones de política CRiminal}

Una de las principales cuestiones político-criminales relacionada con la problemática de la autonomía universitaria es si resulta necesaria y conveniente la promulgación de una legislación estatal que regule esta materia. Esta ha sido la opción en Estados Unidos ${ }^{30}$. Una regulación estatal tiene como principal ventaja el aseguramiento de un estándar mínimo de protección en todas las universidades chilenas. Como desventaja pueden señalarse dos: una limitación de la autonomía universitaria y una menor flexibilidad a la hora de regular estos protocolos de acuerdo con la realidad y el contexto social de cada universidad ${ }^{31}$. En todo caso, la promulgación de una ley solo debería contemplar

${ }^{28}$ CARO, 2018.

${ }^{29}$ Bernasconi, 2015 , p. 2.

${ }^{30}$ Estados Unidos cuenta con dos leyes federales que regulan esta problemática. La primera de ellas es la denominada Clery Act (Jeanne Clery Disclosure of Campus Security Policy and Campus Crime Statistics Act). Esta ley, tras varias reformas, requiere a las universidades hacer público un conjunto de delitos, entre los que destacan los relacionados con la violencia sexual, implementar políticas de prevención y la violencia sexual doméstica y de pareja y el acoso (stalking). Véase, Wies, 2015.La segunda ley federal es la denominada Title IX (Title IX of the Education Amendments Act of 1972). Esta ley fue promulgada en su inicio para hacer efectiva la igualdad de oportunidades entre hombres y mujeres en todos los programas de educación que reciben fondos públicos. Con posterioridad, esta ley ha ido incluyendo otras medidas, entre la que destaca la implementación de políticas y protocolos acerca de violencia y acoso sexual (harassment). Debemos destacar que esta ley contempla la posibilidad de restringir o eliminar los fondos públicos en el caso de incumplimiento de estas obligaciones. También, debe señalarse el tratamiento diferenciado de la violencia sexual y el acoso del establecido en el sistema de justicia penal. WIEs, 2015. Junto con estas dos leyes, debe tenerse presente un documento enviado por el presidente de la Oficina de Derechos Civiles del Departamento de Educación, Russlynn Ali, en 2011, a todas las universidades, poniendo de relieve el problema de la violencia sexual en las universidades estadounidenses. Esta carta recuerda que, de acuerdo con el Título IX, las universidades están obligadas a llevar a cabo de manera inmediata y adecuada políticas y medidas para prevenir, investigar y sancionar la violencia sexual con independencia del sistema de justicia criminal. La propia carta detalla algunas de estas medidas como la designación de un coordinador o responsable para recibir y tramitar las denuncias, la provisión de un formulario de denuncia o el establecimiento de un plazo razonable para resolverlas, entre otras. Además, este documento entiende que el procedimiento disciplinario debe regirse por un estándar probatorio de evidencia preponderante más que por los estándares de clara evidencia o más allá de toda duda razonable. Ali, 2011.

31 En este sentido, el TC ha reconocido la potestad del legislador para regular materias o actividades reconocidas prima facie, dentro del ejercicio de la autonomía universitaria. Tribunal Constitucional, 21.06.2013, rol $\mathrm{N}^{\circ} 2487-2013$, considerando $47^{\circ}$. 
los principios fundamentales de garantía, protección y reinserción, las etapas del procedimiento y las principales instituciones.

El Reglamento UACH, al igual que otros protocolos de prevención y sanción de la violencia entre estudiantes, puede reconstruirse a partir de los principales modelos desarrollados en la política criminal.

En cuanto al modelo garantista, deben destacarse el reconocimiento de ciertas garantías procesales de los denunciados y una especial preocupación por el principio de proporcionalidad. Así, el Reglamento UACH cuenta, como posibles soluciones jurídicas, desde una línea de acción, en el caso de que el hecho denunciado no sea constitutivo de violencia, acoso o discriminación, pero que pudiera atentar con la sana convivencia de la comunidad universitaria (Art. 13 b), hasta un elenco de sanciones (amonestación verbal o escrita, pérdida de beneficios sociales o académicos otorgados por la universidad, suspensión de la matrícula de uno a cuatro semestres y su cancelación (Art. 22). Además, el propio Reglamento UACH reconoce expresamente el principio de proporcionalidad como unos de los criterios fundamentales a la hora de aplicar las medidas de protección y las sanciones citadas. Así, respecto de estas últimas, establece como baremos de ponderación: la gravedad del hecho, el daño causado y la eventual concurrencia de agravantes y atenuantes (Art. 22, inc. $2^{\circ}$ ).

El Reglamento UACH también recoge varias medidas inspiradas en un modelo bienestarista. Así, encontramos medidas de carácter resocializador y relacionadas con la justicia restaurativa y procedimental ${ }^{32}$. En cuanto a las primeras, debemos señalar

${ }^{32}$ La razón de incluir las denominadas justicia procedimental y restaurativa dentro del paradigma o modelo bienestarista se debe a que, en último término, lo que se pretende es que con una mayor participación de las partes involucradas se resuelva de mejor manera el conflicto social que subyace, en nuestro caso, en el AVD. La justicia restaurativa y procedimental constituyen las dos principales dimensiones de la denominada justicia organizacional que trata de ofrecer un diagnóstico y respuesta a las percepciones de insatisfacción tanto de la víctima como del victimario en una determinada organización. GREENBERG, 1987. Ambas dimensiones resultan importantes tanto para las víctimas como para los denunciados o sancionados. La justicia distributiva pone el foco de atención en la legitimidad o justicia del resultado. Las víctimas valoran la necesidad y eficiencia de la solución entregada por la organización, mientras que para los denunciados y sancionados lo relevante es la igualdad y proporcionalidad de esta. HARPer et al., 2017, p. 310. Una de las tesis especialmente contraintuitiva mantenida por la academia estadounidense es que las víctimas demandan resultados con finalidades instrumentales (preventivas, simbólicas, etc.) más que retributivas. HARPER et al., 2017, p. 311. En cuanto a la igualdad y la proporcionalidad de las sanciones, los estudios realizados en Estados Unidos y el creciente número de demandas ante la justicia ponen en evidencia que constituye uno de los principales problemas de los protocolos de AVD. Miller, 1999. En cuanto a la justicia procedimental, esta se centra en el proceso a la hora de legitimar un determinado medio o instrumento de control social. Así, mantiene como una de sus principales tesis que los implicados se encuentran más predispuestos a aceptar una determinada decisión, aunque sea desfavorable, cuando el proceso establecido es considerado como justo o legítimo. Suelen establecer cuatro indicadores que inciden en la percepción de un procedimiento legítimo: (a) mecanismos de información y diálogo, (b) respecto, (c) neutralidad y (d) confianza respecto de los operadores del instrumento de control social. Tyler, 2006, pp. 19-40. Además, como manifiesta Frazier (2000), estos indicadores afectan en el tiempo y la manera en que las víctimas se recuperan de la vulneración de sus derechos, así como en las tasas de AVD no detectadas, ya que aquellas se encuentran más predispuestas a denunciar. Ullman y Filipas, 2001, pp. 4-6. En último término, se propone respecto de los protocolos de AVD un cambio estructural en el procedimiento: sustituir el modelo pseudoadversarial por 
las líneas de acción, ya que constituyen un instrumento no solo para evitar conductas que pueden afectar a la sana convivencia de la universidad, sino también para prevenir conductas sancionables por el Reglamento $\mathrm{UACH}^{33}$. Respecto de las sanciones, debe destacarse la de suspensión de la matrícula sujeta a condición. Así, esta sanción pretende que el estudiante sancionado realice algún tipo de actividad o tratamiento destinado a mejorar su bienestar mental y prevenir en el futuro otras acciones de AVD. También debe mencionarse que las medidas de acompañamiento pueden aplicarse también en beneficio de la persona denunciada (Art. 17).

En cuanto a la justicia distributiva, se contempla la posibilidad de proponer una mediación previamente consentida por las partes (Art. 24). Por último, junto con la "Política de prevención y sanción del Acoso, Violencia y Discriminación en la Comunidad Universitaria”, se establecen varios mecanismos que facilitan que las partes involucradas y la comunidad universitaria perciban que los organismos encargados de aplicar el Reglamento UACH respetan sus derechos y los principios de objetividad, igualdad y justicia. La Secretaría juega, en este sentido, un papel fundamental, ya que tiene atribuida la función de comunicación con las partes involucradas (Art. 8), pero además ha asumido tareas de difusión y coordinación con la comunidad universitaria. Así, podemos destacar programas de difusión del Reglamento UACH y reuniones de trabajo con las diferentes instituciones, representantes de los estudiantes y trabajadores de la Universidad.

No obstante, aunque no existen estudios empíricos en Chile, se puede percibir un cierto descontento tanto por parte de víctimas y asociaciones feministas como de los denunciados y sancionados ${ }^{34}$. Pero, en sentido contrario, podemos encontrar posiciones que mantienen que las universidades mediante estos protocolos se encuentran en una mejor situación a la hora de cumplir los objetivos y fines de la justicia restaurativa y procedimental $^{35}$.

En cuanto al modelo procesal, el reglamento puede ser definido como un sistema o procedimiento seudoadversarial. Así, si bien se encuentran garantizados derechos procesales básicos, el Reglamento UACH no contempla algunas de las garantías reconocidas

uno estructurado en la justicia distributiva y procedimental. HARPER et al., 2017, p. 312. Si bien algunos protocolos contienen mecanismo de mediación, su estructura continúa siendo pseudo-adversarial por lo que, para los partidarios de este modelo político-criminal, los problemas de revictimización y reparación del daño y prevención y resocialización del estudiante sancionado continúan sin resolverse satisfactoriamente. MCGLYNN et al., 2012. Así y por lo que respecta a las víctimas, estas necesitan contar su experiencia dramática, observar el arrepentimiento del ofensor, recibir apoyo y participar en la resolución de la vulneración de sus derechos. Harper et al., 2017, p. 312, véase doctrina citada.

${ }^{33}$ Respecto de las denuncias realizadas, $50 \%$ fueron admitidas, $41 \%$ no fueron admitidas, pero vinieron acompañadas por una línea de acción y $9 \%$ no fueron admitidas sin ninguna medida de este tipo. Las líneas de acción han consistido, principalmente, en entrevistas personales con la persona denunciada (14\%), mediación (22\%), actividades de difusión (14\%) y cartas a la comunidad universitaria (50\%). MEmORIA, 2016-2018.

${ }^{34}$ Véase en Estados Unidos, Tyler, 2006.

${ }^{35}$ Chmielewski, 2013 , p. 149. 
en el derecho penal, entre las que destacan los principios de contradicción, inmediación, doble instancia y el estándar probatorio más allá de toda duda razonable.

\section{La competencia territorial de los protocolos AVD}

Una cuestión de especial relevancia político-criminal radica en la determinación de la competencia territorial de estos protocolos y, en especial, de aquellos supuestos de AVD que concurren fuera de las instalaciones universitarias o del contexto de actividades académicas. Por tanto, el asunto a debatir, desde una perspectiva político-criminal, es si estos protocolos pueden constituirse en medios de control social para prevenir y sancionar conductas, muchas de ellas, tipificadas penalmente. Esta problemática, como era de esperar, ha tenido su reflejo en los tribunales de justicia ${ }^{36}$.

Precisamente, una taxonomía de los casos de AVD especialmente provechosa es aquella que se centra en el ámbito de competencia territorial. Así, podemos diferenciar los siguientes grupos de casos:

1. AVD dentro de las instalaciones de la Universidad, por ejemplo, una agresión en el contexto de una paralización o toma universitaria

2. AVD fuera de las instalaciones de la universidad en el contexto de una actividad académica, por ejemplo, una violación con ocasión de unas clases prácticas de estudiantes de ingeniería forestal en un bosque.

3. AVD fuera de las instalaciones de la universidad en el contexto de una actividad extraacadémica organizada o financiada por alguna institución de la universidad, por ejemplo, un abuso sexual con ocasión de una fiesta organizada por un centro de estudiantes.

4. AVD fuera de las instalaciones de la universidad en el contexto de una actividad extraacadémica organizada o financiada informalmente por estudiantes universitarios, por ejemplo, un abuso sexual en una cabaña arrendada por estudiantes con ocasión de una fiesta universitaria.

5. AVD fuera de las instalaciones de la universidad y del contexto de actividades extraacadémicas formales ni informales, en los que sus efectos sobre la víctima se reproducen en la universidad, por ejemplo, un caso de violencia intrafamiliar en el que, con posterioridad, la víctima y victimario ingresan a la misma carrera y curso académico.

6. AVD fuera de las instalaciones de la universidad y del contexto de actividades académicas o extraacadémicas formales o informales, en el que no se derivan ningún efecto en la universidad, por ejemplo, unos abusos sexuales realizados con ocasión de un encuentro casual entre dos estudiantes pertenecientes a dos campus de la Universidad.

36 Respecto del Reglamento UACH, véanse los siguientes recursos de protección: Corte Suprema, 1.07.2019, rol No 5453-2019; Corte de Apelaciones de Valdivia, 22.10-2018, rol No 1870-2018;09. 2019, rol No 1284-2019; 26.07. 2019, rol No 1480-2019; y 13.08. rol No 1677-2019. 
Los casos 1 y 2 no plantean dudas acerca de la competencia territorial de los protocolos. Respecto del caso 6 las universidades no tendrían competencia para sancionar las conductas, pero sí para aplicar medidas de acompañamiento. Así, los casos más problemáticos y problematizados por los tribunales de justicia son los 3,4 y 5 .

Varias son las razones que justifican que estos protocolos conozcan casos más allá de los acaecidos en sus instalaciones o en el contexto de actividades académicas.

La primera es de orden victimológico. Si tenemos presente que la mayoría de los casos de AVD se relacionan de alguna manera con la violencia machista o sexual contra las mujeres, el establecimiento de una regla de competencia territorial delimitada solo a las instalaciones y actividades de las universidades dejaría fuera de este tipo de protección a un gran número de mujeres. A esto, debemos mencionar la falta de protección por parte de las instituciones estatales debido, entre otras razones, al sistema de garantías y estándar de prueba en el proceso penal y a la falta de recursos.

La segunda razón es de orden político. Un Estado democrático demanda la participación de todos los ciudadanos e instituciones en la prevención y sanción de AVD. Así, los casos que, de alguna manera, afectan al desarrollo académico y a la salud de los estudiantes justifica la intervención activa de las universidades.

Debe advertirse, antes de todo, que la concurrencia de una responsabilidad disciplinaria junto con una eventual pena no genera ningún de tensión con el principio ne bis in idem. En primer lugar, el fundamento de ambas es diferente: la responsabilidad penal se centra en la protección de bienes jurídicos de especial relevancia social (la integridad física, la libertad sexual, etc.) y la responsabilidad disciplinaria pretende asegurar, en último término, por medio de las sanciones, medidas de protección y acompañamiento, unas condiciones básicas para el ejercicio del derecho a la educación ${ }^{37}$.

Como hemos mencionado, la autonomía universitaria ha permitido que las universidades dicten protocolos destinados a prevenir y sancionar este tipo de conductas. En efecto, estas no deben configurarse al margen de las relaciones de poder que caracterizan a la sociedad chilena. La autonomía universitaria debe garantizar no solo la libertad que toda comunidad necesita para el cumplimiento de sus fines y funciones en el ámbito de la docencia, la investigación y la extensión, sino también la formación ciudadana de sus estudiantes. A lo anterior, debemos añadir su necesaria contribución al desarrollo del país y de la sociedad civil $^{38}$.

${ }^{37}$ Recordemos que el Reglamento UACH en su art. 15, al igual que ocurre con la potestad disciplinaria de la Administración, establece que la terminación del proceso penal sin condena (sobreseimiento, suspensión condicional, absolución, etc.) no cierra la posibilidad de que el hecho sea sancionado por el Reglamento UACH.

38 Por todos, Ávila y Guillezeau, 2010, p. 175. La Declaración de Guadalajara sobre Autonomía Universitaria (mayo de 2011) de la Unión de Universidades de América Latina (UDUAL) contempla una visión de la autonomía universitaria más integral: "La Universidad se enmarca en un amplio conjunto de prerrogativas fundamentales, entre las cuales se destacan la libertad de pensamiento y la libertad de cátedra para la generación de conocimiento tendiente a la formación integral de la persona y el desarrollo democrático de la sociedad". Negrita nuestra. 
Dentro de las críticas en contra de estas reglas de extensión de la potestad disciplinaria de las universidades, podemos destacar dos. La primera se centra en los límites de la autonomía universitaria.

La Corte Suprema en un litigio, precisamente, de competencia del Reglamento $\mathrm{UACH}$, ha establecido una suerte de interpretación orientada a la Constitución de esta regla especial de competencia. Así, sostiene que "los tribunales de justicia han reconocido con carácter general, de acuerdo con la letra a) del artículo $2^{\circ}$ de la Ley $\mathrm{N}^{\circ} 21.091$ sobre Educación Superior, la autonomía de las universidades para regular mediante su regulación interna este tipo de actividades, siempre que esta potestad disciplinaria se oriente a sus fines y proyectos institucionales"39. A partir de esta consideración de tipo funcionalista, la Corte Suprema ha establecido una interpretación restrictiva de la regla excepcional de competencia del citado reglamento. Así, mantiene que no basta la mera "circunstancia de estar involucrados en los hechos personas relacionadas con la universidad por algún vínculo docente o funcionario o de otra naturaleza análoga, porque, precisamente, las potestades que derivan de la autonomía universitaria se extienden, como lo dispone la norma transcrita, hasta donde alcancen sus fines y proyectos institucionales" ${ }^{40}$. En otras palabras, se requiere además de una vinculación subjetiva (la condición de estudiantes universitarios) una objetiva relacionada con los fines de la universidad. De esta manera, la Corte Suprema incluye una nueva condición de aplicación, en principio, extensible a todos los protocolos de las universidades chilenas con independencia de las concretas reglas de competencia establecidas por cada una de ellas.

La cuestión problemática de este razonamiento es que de facto puede dejar sin contenido este tipo de reglas de competencia, ya que, como hemos mencionado, se dejaría fuera de protección un número considerable de casos graves (violencia en el pololeo y abusos o agresiones sexuales realizadas en el contexto de fiestas universitarias).

Una interpretación alternativa a esta comprensión de la Corte Suprema es entender que existen otras conexiones objetivas más allá de las estrictamente académicas. Efectivamente, las universidades y, en concreto la UACH, no tienen como única función institucional las diferentes manifestaciones de la actividad académica sino que, como actores sociales, persiguen otras funciones, como es precisamente prevenir y sancionar conductas de AVD que, de alguna manera, se vean institucionalmente involucradas. Además, en el caso de la UACH, esta cuenta con una "Política de Prevención y Sanción del Acoso, la Violencia y Discriminación en la Comunidad Universitaria” que, expresamente, declara como contrarios a los principios y valores de la Corporación "todo fenómeno de acoso, violencia y discriminación, incluidos los que ocurren en el marco de

39 Negrita nuestra.

${ }^{40}$ Corte Suprema, 1.07.2019, rol N 5453-2019, Cons. $7^{\circ}$. La Corte Suprema concluye que la aplicación de esta regla excepcional de competencia sobrepasa "los márgenes académicos, por hechos propios de la competencia común de los tribunales establecidos por la ley, con lo cual la recurrida se ha constituido, desde este ángulo, en una comisión especial, como quiera que carece de origen legal” (Cons. $11^{\circ}$ ). Véanse las sentencias de la Corte de Apelaciones de Valdivia de 26 de julio de 2019, Rol N 1480-2019, Cons. 7 y la sentencia de la Corte Suprema, Rol 33389, 22.05.2019. 
relaciones de pareja" ${ }^{41}$. Incluso se puede entender que la prevención y sanción de estas conductas constituyen un presupuesto para que las universidades puedan llevar a cabo sus tareas académicas. Así, resulta difícil que, por ejemplo, una víctima de AVD ocasionado en el contexto de una relación de pareja con otro estudiante o de una actividad lúdica pueda desarrollar sus actividades académicas de manera adecuada, si debe convivir con el agresor en las instalaciones universitarias. En otras palabras, esta regla de competencia territorial garantiza, en último término, una igualdad material en la educación ${ }^{42}$. Y como estamos hablando de derechos fundamentales, debemos recalcar, de acuerdo con la criminología crítica y la teoría feminista, que razonamientos formales, como la delimitación del protocolo a actividades estrictamente académicas, no puede constituir un criterio necesario y suficiente, ya que excluye de facto no solo los principales casos de AVD, sino que también limita o impide el derecho a la educación a sus víctimas ${ }^{43}$.

La segunda crítica o cuestionamiento es de tipo institucional y económico. Las universidades complejas cada vez están asumiendo mayores atribuciones en políticas sociales debido, entre otras razones, al modelo de Estado chileno. Así, por ejemplo y relacionado con AVD, la problemática de la salud mental de los estudiantes constituye en la actualidad uno de los temas principales de la agenda política universitaria. El problema es que, al igual que el AVD, los establecimientos universitarios no tienen la capacidad económica e institucional para abordar todos los problemas de salud mental de los estudiantes, más cuando una parte considerable de estos tiene sus causas fuera de las aulas universitarias.

En todo caso, lo relevante no es tanto dirimir si deben ser las instituciones del Estado, las universidades o ambas en conjunto las que deben conocer de los casos de AVD, sino garantizar una adecuada respuesta de acuerdo con el Estado social de derecho.

\section{El reconocimiento de los derechos de las víctimas: justicia restaurativa y resocialización versus punitivismo (feminista)}

Sin duda, la principal novedad en materia político-criminal del Reglamento UACH tiene relación con aquellas medidas que tienen como finalidad garantizar y reconocer los derechos de las víctimas.

Las víctimas se han convertido en los últimos tiempos en un actor principal en la política criminal. Mediante su organización y de una creciente atención por parte de los

${ }^{41}$ Decreto $\mathrm{N}^{\mathrm{0}} 76,2015$, p. 4.

42 No resulta muy difícil de concluir que los casos de AVD generan graves efectos psicológicos que pueden impedir a la víctima continuar con sus estudios universitarios. CHMIELEwski, 2013, pp. 165-167.

${ }^{43}$ Esta interpretación, en el caso del Reglamento UACH, requiere adecuarse a la norma especial de competencia establecida en su Art. 2 que, recordemos, establece como requisitos para conocer casos de AVD fuera de las instalaciones de la universidad y del contexto de actividades académicas, la gravedad y necesidad de protección de la víctima. Si bien una reforma del Reglamento UACH resulta la solución más adecuada, entiendo que esta interpretación resulta compatible, ya que nos encontraríamos ante una interpretación restrictiva de dicha norma especial de competencia. Así, junto con estos dos requisitos, el AVD debe orientarse a sus fines y proyectos institucionales tanto académicos como sociales. 
medios de comunicación, se ha conseguido llamar la atención de los poderes e instituciones públicas, lo que, a la vez, ha derivado en una de las principales manifestaciones de la juridificación de los nuevos procesos de inclusión.

El reconocimiento de los derechos de las víctimas puede incluirse tanto el modelo de seguridad ciudadana o punitivista, como en el modelo bienestarista o no punitivista, asociado este último, especialmente, a la justicia restaurativa.

El modelo punitivista -heredero de una visión retributiva, preventiva (general y especial negativa) o expresiva de la pena- propone, como mecanismo de reconocimiento de los derechos de las víctimas un mayor rigorismo penal, la inclusión de ciertos derechos procesales a favor de estas y una restricción de los derechos y garantías penales del acusado o condenado. Así, para este modelo, el sistema penal opera como un juego de suma cero, en el que cualquier ganancia de la víctima debe ser a expensas del acusado ${ }^{44}$. A la vez, se cuestiona la visión garantista a favor de la no punibilidad de conductas consideradas libremente consentidas, como la prostitución o la pornografía. Incluso, se muestra contraria a ciertas formas de justicia distributiva, como ocurre en nuestro caso con los acuerdos reparatorios ${ }^{45}$.

También puede utilizar técnicas populistas, como son las movilizaciones sociales fundamentadas en casos trágicos (sobre) divulgados por los medios de comunicación ${ }^{46}$, creando una insatisfacción continua de las víctimas y de la sociedad en general. No obstante, el populismo penal y, en concreto, el de corte feminista, no debe ser visto, en todo caso, como un fenómeno socialmente negativo ${ }^{47}$. Sin duda, la aprobación de los protocolos de prevención y sanción de la violencia en las universidades se debe en gran parte en la repercusión mediática que tuvieron las protestas estudiantiles de 2018.

En el mismo sentido, puede comprobarse una actitud de tolerancia cero y, especialmente, punitivista ante este tipo de conductas. Así no resulta infrecuente que asociaciones estudiantiles que denuncian la vulneración de principios penales -como el de proporcionalidad de las penas y el de presunción de inocencia- respecto de la aplicación de la legislación antiterrorista a los miembros de la comunidad mapuche, adopten, por el contrario, posturas punitivistas y de intolerancia respecto del acoso sexual en los campus universitarios. Sin duda, los grupos de presión relacionados con los grupos feministas han logrado que se aprueben varias medidas contra hechos que, hasta tiempos recientes, eran tolerados e invisibilizados por el Estado y la comunidad, pero si estas se aplican no en función de la entidad de la conducta, sino principalmente en relación con quién

${ }^{44}$ LOADER, 2008, p. 3.

45 Art. 19 de la Ley No 20.066 sobre Violencia Intrafamiliar.

${ }^{46}$ Un ejemplo paradigmático de esta situación ocurrió en la difusión que algunos medios de comunicación estadounidenses dieron a un estudio del Instituto Nacional de Justicia, en el que se concluyó que una de cada cinco estudiantes universitarias habían sido objeto de una agresión sexual. Dentro del concepto de agresión sexual establecido en el estudio se incluían conductas que iban desde la violación con violencia o intimidación a un tocamiento no consentido. Algunos medios informaron que una de cada cinco estudiantes universitarias había sido víctima de una violación. BEAVER, 2017: 258-259.

47 Veáse, en general, LACLAU, 2016. 
sea la víctima o el victimario o como medio para la consecución de una agenda política, estas pueden derivar en prácticas discriminatorias. En este sentido, debe tenerse presente que parte de la denominada victimología positivista y algunos movimientos feministas comparten la crítica al modelo bienestarista clásico por centrar sus medidas y políticas públicas en la resocialización del delincuente ${ }^{48}$.

Por el contrario, el modelo no punitivista de reconocimiento de los derechos de las víctimas trata de minimizar los efectos indeseados de la violencia con medidas centradas en la prevención y la justicia restaurativa ${ }^{49}$. Así, esta forma de reconocimiento de las víctimas se centra en algunas políticas destinadas a proteger a las más vulnerables, donde la familia y la comunidad juegan un papel fundamental. Además, comparte con el garantismo el escepticismo respecto de la utilidad de la pena ${ }^{50}$.

El Reglamento UACH, en general, puede encuadrase dentro de un modelo de reconocimiento de las víctimas no punitivista, aunque en lo referido a las garantías procesales, algunas de las restricciones que contiene pueden encuadrarse en un modelo punitivista.

Sin duda, las medidas del Reglamento UACH que han tenido un mayor impacto en el reconocimiento de los derechos de las víctimas son las de protección (Art. 16) y acompañamiento (Art. 17). En cuanto a las primeras, tanto la Comisión como la Secretaría han establecido varias medidas con la finalidad de otorgar asistencia médica o psicológica, asesoramiento legal y apoyo social en beneficio de las víctimas ${ }^{51}$.

Las medias de protección que, en general, comparten la misma finalidad y fundamentación que las medidas cautelares, constituyen mecanismos de reconocimiento de los derechos de las víctimas, aunque tienen también una finalidad preventiva (Art. 16). Estas medidas comprenden desde la prohibición de contacto entre las partes implicadas hasta la restricción total o parcial del denunciado a las instalaciones de la universidad ${ }^{52}$. En todo caso, el citado artículo establece expresamente la obligatoriedad de la UACH de asegurar el derecho constitucional a la educación del denunciado con medidas tales como su traslado a cursos paralelos o tutorías personalizadas.

Otra medida relevante es la posibilidad de que la Comisión puede nombrar de oficio, en casos de especial vulnerabilidad, un representante de la víctima (Art. 18).

${ }^{48}$ Véase en extenso, Davies, 2017, pp. 12-40, 82-90. En este sentido debemos mencionar la siguiente reflexión de Segado: "No quiero un feminismo del enemigo, porque la política del enemigo es lo que construye el fascismo (...). El feminismo punitivista puede hacer caer por tierra una gran cantidad de conquistas [es] un mal sobre el que tenemos que reflexionar más: ¿Puede un estado con las cárceles hacer justicia? Esa no puede ser la justicia; ser justo con una mano y ser cruel con la otra (SEGATO, 2018).

49 ROACH, 1999, pp. 673 y 669.

${ }^{50}$ ROACH 1999, pp. 670

51 Dentro de estas medidas, destaca la asistencia psicológica (91\%), medidas de flexibilización académica (33\%) y orientación jurídica (5\%). Un 26\% se aplicaron conjuntamente las medidas de asistencia psicológica y flexibilización académica (Memoria, 2016-2018).

${ }^{52}$ Las medidas de protección se concretaron, principalmente, en la prohibición de contacto entre las partes implicadas (62\%), tutorías sustitutivas (14\%), restricción a actividades lectivas (9\%) y restricción parcial o total al campus ( $5 \%$ ). Debe tenerse presente que estas medidas pueden aplicarse de manera conjunta. 
También encontramos, como hemos mencionado, una regla ponderativa reforzada a favor de los derechos de las víctimas (art. 11, e $)^{53}$.

Por último, en la determinación de las condiciones aplicables en la sanción de suspensión de la matrícula, se presta especial resguardo en asegurar el normal desarrollo de las actividades académicas y universitarias de la víctima. Especialmente relevante es la medida que restringe al sancionado el acceso a ciertos lugares en los que podría coincidir con esta (Art.23).

\section{A MOdo DE CONCLUSIÓN}

Como hemos mencionado, la finalidad de este estudio es contribuir al conocimiento de los protocolos AVD a partir de una reconstrucción desde la criminología y la política criminal. De esta manera, la comunidad universitaria, en su caso, los operadores jurídicos y la ciudadanía en general pueden entender y criticar con mejores fundamentos la estructura, principios y lógicas de estos instrumentos.

A partir de un modelo bienestarista, el estudio propone un papel más activo de las universidades a la hora de sancionar y prevenir la violencia mediante una extensión de su competencia territorial y un reconocimiento de los derechos tanto de las víctimas, con medidas de protección y acompañamiento y soluciones alternativas, como de los estudiantes mediante un debido proceso, una sanción proporcionada y unas medidas de reinserción.

\section{BIBLIOGRAFÍA}

Abdelkader, José Miguel, 2011: Modelos de seguridad y policía en campus universitarios, Valencia: Tirant lo Blanch.

Ali, Russlynn, 2011: Dear Colleague Letter. Office for Civil Rights, April 4. Disponible: http:// www2.ed.

Ávila, Nelson, y Gillezeau, Patricia, 2010: “Autonomía universitaria y su misión transformadora: Enfoque teórico-histórico”, Revista de Ciencias Sociales, Vol. XVI, 1.

Ballbé, Manuel, 1999: Dictamen sobre la autonomía universitaria y el mantenimiento del orden académico en el campus, Universidad Autónoma de Barcelona.

Bassa, Jaime y Aste Leiva, Bruno, 2019: “Autonomía universitaria: Configuración legislativa de su contenido constitucional”, Estudios constitucionales, 17 (1).

Beaver, William R.: "Campus Sexual Assault: What We Know and What We Don't", The Independent Review, 22 (2).

Bernasconi, Andrés, 2015: “Autonomía Universitaria en el Siglo XXI: nuevas formas de legitimidad ante las transformaciones del Estado y la Sociedad”, Páginas De Educación, 7 (2).

\footnotetext{
${ }^{53}$ Esta medida puede considerarse también en un sentido amplio como punitivista.
} 
Caro, Cindy J., 2018: "Las sobrevivientes de acoso sexual universitario en Colombia siguen unidas y resistiendo", VICE Colombia. Disponible: https://www.vice.com/amp/es_latam/ article/j5zx4y/las-victimas-de-acoso-sexual-universitario-se-resisten-a-serlo-para-siempre

Chmielewski, Amy, 2013: "Defending the Preponderance of the Evidence Standard in College Adjudications of Sexual", Brigham Young University Education and Law Journal, 3-1.

Fisher, Bonnie S. et al., 2000: The Sexual Victimization of College Women, Washington, DC: NIJ- BJS. Davies, Pamela et al., 2017: Victims, Crime and Society. An introduction (2 ${ }^{\mathrm{a}}$ ed.), Sage Publications. Fernández, José A., 2019: "Caso del 'beso forzado'”, Casos destacados. Derecho penal, Parte especial, Vargas, Tatiana (Dir.).Frazier, Patricia A., 2000: "The role of attributions and perceived control in recovery from rape", Journal of Personal and Interpersonal Loss, 5.

Greenberg, Jerald, 1987: “Taxonomy of organizational justice theories", Academy of Management Review, 12 (1).

Harper, Shannon et al., 2017: "Title IX Due Process Standards in Campus Sexual Assault Adjudication: Considering the Roles of Distributive, Procedural, and Restorative Justice", Journal of School Violence, 16 (3).

Koss, Mary P. et al., 1987: "The scope of rape: Incidence and prevalence of sexual aggression victimization in a national sample of higher education students", Journal of Consulting and Clinical Psychology, 55.

LaClau, Ernesto, 2016: La razón populista, Madrid: Fondo De Cultura Económica de España.

Levesque, Deborah A. et al., 2016: "Teen Dating Violence Prevention: Cluster-Randomized Trial of Teen Choices, an Online, Stage-Based Program for Healthy, Nonviolent Relationships”, Psychological Violence, 6.

Lisak, David. y Miller, Paul. M. 2002: "Repeat Rape and Multiple Offending Among Undetected Rapists”, Violence and Victims, 17 (1).

LoAder, Ian, 2006: "Fall of the 'Platonic Guardians': Liberalism, Criminology and Political Responses to Crime in England and Wales", British Journal of Criminology, 46 (4).

Mcglynn, Clare et al., 2012, “' I Just Wanted Him to Hear Me': Sexual Violence and the Possibilities of Restorative Justice", Journal of Law and Society, 39 (2).

Memoria de la Comisión para la Intervención en Situaciones de Acoso, Violencia y DiscriminaCión ENTRE ESTUdiantes, 2016-2018 (pendiente de publicación).

Poó, Ana María. y Vizcarra, María Beatriz, 2008: "Violencia de Pareja en Jóvenes Universitarios”, Revista Terapia Psicológica, 26 (1).

Martin, Patricia Y. y Hummer, Robert. A, 1989: "Fraternities and rape on campus", Gender E Society, 3 (4).

Mengo, Cecilia. y Black, Beverly. M., 2016: "Violence Victimization on a College Campus: Impact on GPA and School Dropout", Journal of College Student Retention, 18 (2).

Mellins, Claude A. et al., 2017: "Sexual assault incidents among college undergraduates: Prevalence and factors associated with risk", Plos one, 12 (11).

SEgATO, Rita, 2018:"El feminismo punitivista puede hacer caer por tierra una gran cantidad de conquistas", Agencia Paco Urondo. Periodismo Militante. Disponible: http://www.agenciapacourondo.com.ar/ generos/rita-segato-el-feminismo-punitivista-puede-hacer-caer-por-tierra-una-gran-cantidad-de

TripletT, Matthew R., 2012: "Sexual assault on college campuses: Seeking the appropriate balance between due process and victim protection", Duke Law Journal, 62 (2).

Tyler, Tom R., 2006: Why the people obey the law, New Jersey: Prince University Press.

Ullman, Sarah E. y Filipas, Henrietta H., 2001: "Predictors of PTSD symptom severity and social reactions in sexual assault victims", Journal of Traumatic Stress, 14 (2). 
Perkins, Wendy y Warner, Jessica, 2017: "Sexual Violence Response and Prevention: Studies of Campus Policies and Practices", Journal of School Violence, 16: (3).

Wies, Jennifer R., 2015: "Title IX and the State of Campus Sexual Violence in the United States: Power, Policy, and Local Bodies", Human Organization, 74 (3).

\section{Jurisprudencia}

Tribunal Constitucional, 21.06.2013, rol No 2487-2013.

Corte Suprema, 1.07.2019, rol No 5453-2019.

\section{Legislación}

LEY N ${ }^{\circ} 20.066,22.09-.005$, que "establece Ley de Violencia Intrafamiliar".

Jeanne Clery Disclosure of Campus Security Policy and Campus Crime Statistics ACT (Public Law 105-244).

Title IX of the Education Amendments Act of 1972 Nº $^{\circ} 92318,86$ Stat. 235, 20 U.S.C. $\S \S 1681-1688$.

Decreto Ley N ${ }^{\circ}$ 50, 1.09.1973, designa Rectores-delegados en Universidades del País.

Universidad Austral de Chile, Decreto No 76, 15-12-2015, que "Aprueba la Política de Prevención y Sanción del Acoso, Violencia y Discriminación en la Comunidad Universitaria”.

Universidad Austral de Chile, Decreto No 28, 22.06.2016, que "Reglamenta Procedimiento para el Acompañamiento, Investigación y Sanción de Conductas de Acoso, Discriminación y Violencia entre Estudiantes de la Universidad Austral de Chile".

Proyecto Modifica Ley de Violencia Intrafamiliar y otros cuerpos legales y establece Ley sobre Violencia en las Relaciones Íntimas de Pareja sin Convivencia, Boletín $\mathrm{N}^{\circ}$ 8851-18. Disponible: https://www.senado.cl/appsenado/templates/tramitacion/index. php?boletin_ini=8851-18. 
